\title{
tacmagic: Positron emission tomography analysis in $\mathrm{R}$
}

\author{
Eric E. Brown ${ }^{1,2}$ \\ 1 Department of Psychiatry and Institute of Medical Science, University of Toronto, Toronto, \\ Canada 2 Centre for Addiction and Mental Health, Toronto, Canada
}

DOI: $10.21105 /$ joss. 01281

\section{Software}

- Review ¿

- Repository ¿

- Archive c

Submitted: 26 February 2019 Published: 27 February 2019

\section{License}

Authors of papers retain copyright and release the work under a Creative Commons Attribution 4.0 International License (CC-BY).

\section{Background}

Positron emission tomography (PET) is a research and clinical imaging modality that uses radioactive tracers that bind to target molecules of interest. A PET scanner identifies the tracer location by virtue of the tracer's radioactive decay, providing information about the distribution of target in the body. Analysis pipelines are used to calculate radiotracer activity over time within a spatial region of interest (ROI). The resulting time-activity curves (TAC) are analyzed to answer important clinical and research questions using kinetic models (Dierckx, Otte, Vries, \& Waarde, 2014).

\section{The tacmagic $\mathbf{R}$ package}

By supporting multiple source data formats, tacmagic provides an open $\mathrm{R}$ ( $\mathrm{R}$ Core Team, 2018) platform for the analysis of PET TAC data that has been produced by existing image analysis pipelines. The data loading functions provide a common format for subsequent analysis in R. We have also implemented basic non-invasive models commonly used in PET research (Logan et al., 1996; Lopresti et al., 2005), which have been tested against existing tools (Oikonen, 2018). The goal is to facilitate open, explicit and reproducible research.

The major features of tacmagic are documented in a walkthrough vignette that is included with the package. The features include:

1. loading TAC and volume data to analyze in R,

2. merging regional TAC data into larger ROIs weighted by volume,

3. basic TAC plotting,

4. calculation of standardized uptake value ratio (SUVR) (Dierckx et al., 2014; Lopresti et al., 2005),

5. calculation and plotting of the non-invasive reference region Logan DVR model (Logan et al., 1996; Oikonen, 2018) and

6. calculation of cut-off values for dichotomizing data (Aizenstein et al., 2008).

The package is published with an open source licence, enabling future collaboration and expansion of the package's functions, which may include future support for additional data formats, kinetic models, plotting and cut-off calculation. 


\section{Acknowledgments}

Many thanks are due to the kind mentorship of Ariel Graff-Guerrero, Philip Gerretsen and Bruce Pollock, as well as to Fernando Caravaggio, Jun Chung, and Tiffany Chow for their guidance in PET analysis techniques prior to the development of this package.

Development of parts of this package involved work supported by the Canadian Institute for Health Research Canada Graduate Scholarship, the Ontario Graduate Scholarship, and the Clinician Scientist Program of the University of Toronto's Department of Psychiatry.

\section{References}

Aizenstein, H. J., Nebes, R. D., Saxton, J. A., Price, J. C., Mathis, C. A., Tsopelas, N. D., Ziolko, S. K., et al. (2008). Frequent amyloid deposition without significant cognitive impairment among the elderly. Archives of Neurology, 65(11), 1509-1517. doi:10.1001/ archneur.65.11.1509

Dierckx, R. A. J. O., Otte, A., Vries, E. F. J. de, \& Waarde, A. van (Eds.). (2014). PET and SPECT in Psychiatry. Springer-Verlag Berlin Heidelberg.

Logan, J., Fowler, J. S., Volkow, N. D., Wang, G.-J., Ding, Y.-S., \& Alexoff, D. L. (1996). Distribution volume ratios without blood sampling from graphical analysis of pet data, 16(5), 834-840. doi:10.1097/00004647-199609000-00008

Lopresti, B. J., Klunk, W. E., Mathis, C. A., Hoge, J. A., Ziolko, S. K., Lu, X., Meltzer, C. C., et al. (2005). Simplified quantification of pittsburgh compound b amyloid imaging pet studies: A comparative analysis. Journal of Nuclear Medicine, 46, 1959-1972.

Oikonen, V. (2018). Tpcclib: Command-line tools to processing and analyzing data collected in turku pet centre. https://gitlab.utu.fi/vesoik/tpcclib; Turku PET Centre, Turku, Finland.

R Core Team. (2018). R: A language and environment for statistical computing. Vienna, Austria: R Foundation for Statistical Computing. Retrieved from http://www.R-project. org/ 